\title{
Facile Synthetic Route Toward High Conversion Primary Aliphatic Poly(vinyl benzyl isocyanate) via Iodination
}

\author{
By Wei-Ho TING, ${ }^{1}$ Shenghong A. DAI, ${ }^{1}$ Hsun-Tsing LEE, ${ }^{2}$ Franklin M. C. CHEN, ${ }^{1,3}$ \\ Wen-Chiung $S U^{4}$ and Ru-Jong JENG ${ }^{1, *}$
}

\begin{abstract}
Aliphatic isocyanato polymers, poly(vinyl benzyl isocyanate)s were synthesized via iodination and isocyanation from starting materials poly(vinyl benzyl chloride)s. This new process does not require phosgene which is highly toxic. This synthetic route takes advantage of the unique property of iodide ion which functions not only as a good nucleophile for iodination but also as a leaving group for isocyanation. The isocyanation conditions are mild with reaction temperatures ranging from 30 to $70{ }^{\circ} \mathrm{C}$. In addition, the process is very efficient with conversions over $90 \%$, yielding no dimer, trimer or other byproducts. KEY WORDS: Poly(vinylbenzyl isocyanate) / Iodination / Isocyanation / TEMPO /
\end{abstract}

Isocyanato functional groups are highly reactive toward amines and alcohols to form urea and urethane compounds, respectively. Extremely large amounts of polyurethanes (PUs) have been produced for daily necessities in various industries. In one example, PUs are used extensively in rigid and soft foams which are widely used in commercial products, and also capable of satisfying specific needs such as adhesives, coatings and sealants. Apart from that, isocyanato polymers have attracted lots of attention because of their wide range of applications. Synthesis of isocyanato polymers that contain polystyrene (PS) backbones falls into two categories.

For the first category, one can directly polymerize the vinylcontaining isocyanato monomer such as m-isopropeny- $\alpha, \alpha^{\prime}$ dimethylbenzyl isocyanate (TMI). ${ }^{1-6}$ However, the TMI monomers do not polymerize to any significant extent via free radical bulk homopolymerization, as addressed by Leonie et $a .^{7}$ The reluctance of TMI monomers to homopolymerize is due to the presence of $\alpha$-methyl group, which causes significant steric hindrance resulting in a substantial reduction of propagation rate coefficient, $k_{\mathrm{p}}\left(\left(60^{\circ} \mathrm{C}\right.\right.$, styrene $)=340$ $\mathrm{L} \mathrm{mol}^{-1} \mathrm{~s}^{-1}$ and $k_{\mathrm{p}}(\alpha$-methyl styrene $\left.)=2.61 \mathrm{~L} \mathrm{~mol}^{-1} \mathrm{~s}^{-1}\right)$. It is concluded that the TMI monomers are not suitable for synthesizing homopolmers or block copolymers.

For the second category, one can initially polymerize functional monomers. Subsequently, the polymers undergo isocyanation to obtain isocynato polymers. For applications, one prefers aliphatic isocyanato polymers to the aromatic ones because aliphatic isocyanato polymers are less likely to discolor and have better shelf life than the aromatic polymers. Himel et al. ${ }^{8}$ patented the reaction of poly(vinyl benzyl chloride) (PVBC) with sodium cyanate at high temperatures using a highly polar solvent as the reaction medium to obtain poly(vinyl benzyl isocyanate) (PVBNCO) polymer. However, this process resulted in low conversion for the PVBNCO polymer, and the production of isocyanurate byproduct. Gaonkar et al. ${ }^{9}$ adopted the same concept to synthesize PVBNCO, but replaced the medium with toluene. In addition, a $\mathrm{S}_{\mathrm{N}} 2$ isocyanation reaction was carried out at a lower temperature under catalytic conditions. The catalyst was used to promote the isocyanation conversion. However, the byproducts, especially trimers and dimers were present in the system, and the conversion of byproducts could reach to levels as high as $16 \%$ in all cases, as displayed in Scheme 1 (route 1).

Based on the above, it is imperative to develop a facile synthetic route toward obtaining aliphatic isocyanato polymers. Wentrup et al. ${ }^{10}$ treated primary alkyl iodide with silver cyanate to produce isocyanato compounds without the addition of any catalyst. It is noteworthy that the byproduct was absent in the isocyanation process. Because of this, Wentrup's approach was used for the synthesis of our polymeric system. In this work, aliphatic isocyanato polymers-poly(vinyl benzyl isocyanate)s was synthesized via iodination and isocyanation from starting materials poly(vinyl benzyl chloride)s instead of the toxic phosgene process, as shown in Scheme 1 (route II). Through the above-mentioned synthetic route, aliphatic isocyanato homopolymer, and random copolymer can be obtained with high conversion.

\section{EXPERIMENTAL}

\section{Materials and Reagents}

Benzoyl peroxide (BPO), 2,2,6,6-tetramethyl-1-piperidinyloxy (TEMPO), sodium iodide (NaI), potassium iodide (KI), sodium cyanate $(\mathrm{NaOCN})$, silver cyanate $(\mathrm{AgOCN})$, styrene (St), 4-vinyl benzyl chloride (VBC), hydroxy ethyl methacrylate (HEMA), acetone and toluene were purchased from Aldrich Co. Prior to use, St and VBC were freshly distilled under reduced pressure.

\footnotetext{
${ }^{1}$ Department of Chemical Engineering, National Chung Hsing University, 250 Kuo-Kuang Road, Taichung 402, Taiwan

${ }^{2}$ Department of Chemical and Materials Engineering, Vanung University, 1 Van-Nuing Road, Tao-Yuan 32061, Taiwan

${ }^{3}$ Department of Natural and Applied Sciences (Chemistry), University of Wisconsin-Green Bay, Wisconsin 54311, USA

${ }^{4}$ Chung-Shan Institute of Science and Technology, Lungtan, Taoyuan 325, Taiwan

*To whom correspondence should be addressed (Tel: +886-4-22852581, Fax: +886-4-22854734, E-mail: rjjeng@nchu.edu.tw).
} 


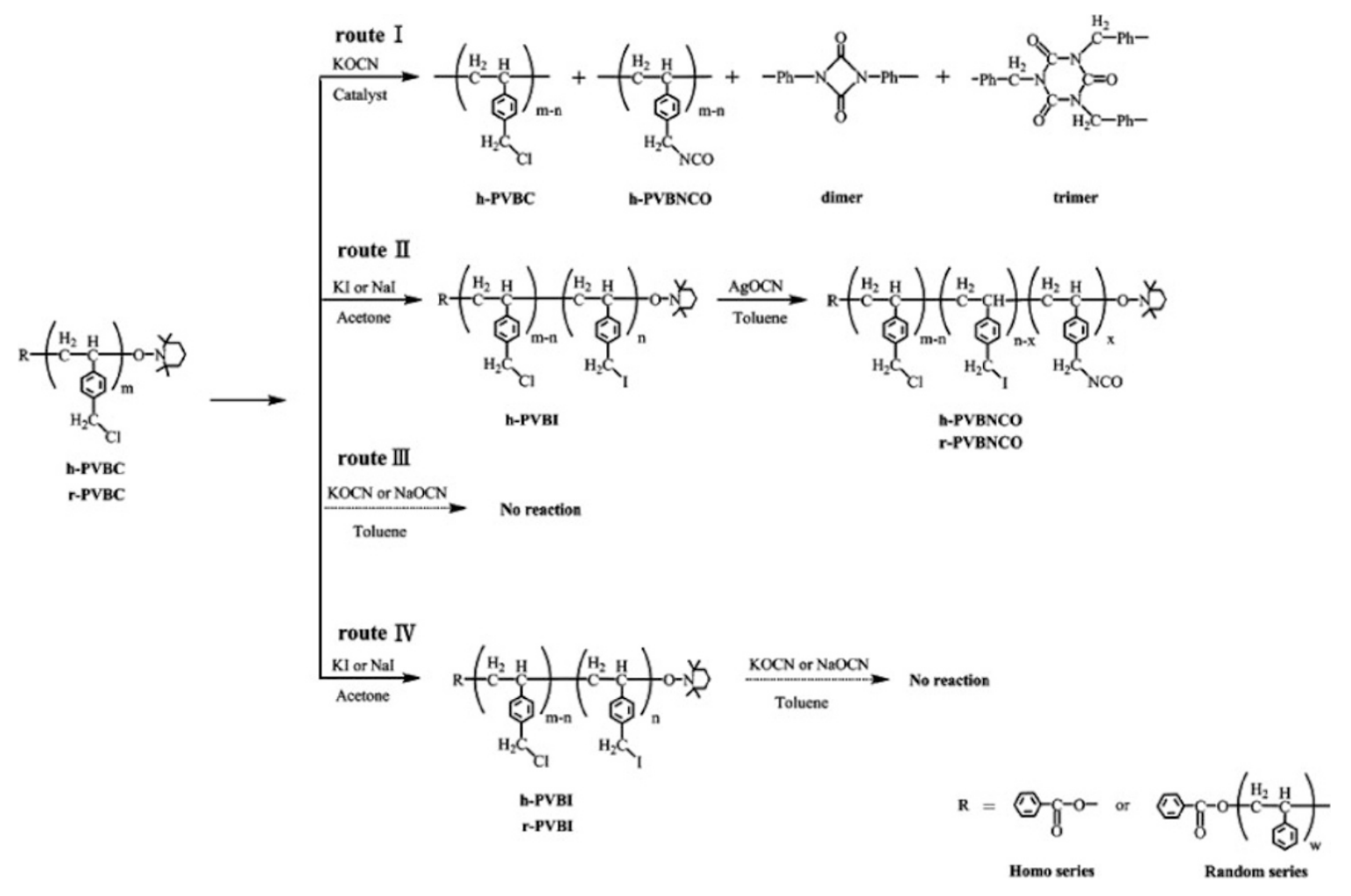

Scheme 1. Synthesis of poly(vinyl benzyl isocyanate).

\section{Synthesis of Polymers}

Preparation of homo-Polymer (h-PVBC). Bulk polymerization was carried on purified commercial grade sample of VBC monomer. Typically, the purified VBC (9.16 g), BPO (56 mg) and TEMPO $(47.7 \mathrm{mg}$ ) was added in $50 \mathrm{~mL}$, three-neck flask. The mixture was heated at reflux temperature $\left(125^{\circ} \mathrm{C}\right)$ for several hours in nitrogen. The polymer, h-PVBC was then purified by repeated precipitations from dichloromethane to methanol. ${ }^{1} \mathrm{H}$ NMR $\left(200 \mathrm{MHz}, \mathrm{CDCl}_{3}, \delta, \mathrm{ppm}\right): 1.10-2.22$ (br, $6 \mathrm{H}$ ), 4.30-4.50 (br, 2H, - $\left.\mathrm{CH}_{2}-\mathrm{Cl}\right), 6.10-7.20$ (br, 9H) ppm. FTIR spectrum $(v)$ : $1266 \mathrm{~cm}^{-1}\left(\mathrm{CH}_{2}-\mathrm{Cl}\right)$. [h-PVBC: Elemental analysis (Found: C, 72.70; H, 6.65\%. requires $\mathrm{C}, 70.82 ; \mathrm{H}$, $5.90 \%) . M_{\mathrm{n}}=12140$, polydispersity $\left.=1.46, T_{\mathrm{g}}=98^{\circ} \mathrm{C}\right]$.

Preparation of Random Copolymer (r-PS-PVBC). Bulk polymerization was carried out on St monomer $(10 \mathrm{~mL})$ and VBC monomer $(10 \mathrm{~mL})$ at $125^{\circ} \mathrm{C}$ for several hours. Subsequently, the random copolymer, r-PS-PVBC was purified by repeated precipitations from dichloromethane into methanol. ${ }^{1} \mathrm{H}$ NMR (200 MHz, $\mathrm{CDCl}_{3}, \delta, \mathrm{ppm}$ ): 1.10-2.22 (br, 6H), 4.304.50 (br, 2H, - $\mathrm{CH}_{2}-\mathrm{Cl}$ ), 6.10-7.20 (br, 9H) ppm. FT-IR spectrum $(v)$ : $1266 \mathrm{~cm}^{-1}\left(\mathrm{CH}_{2}-\mathrm{Cl}\right)$. [r-PS-PVBC: Elemental analysis (Found: C, 86.52; H, 8.31\%. requires $\mathrm{C}, 87.52 ; \mathrm{H}$, $7.29 \%) . M_{\mathrm{n}}=17885$, polydispersity $\left.=1.21, T_{\mathrm{g}}=102^{\circ} \mathrm{C}\right]$.

\section{Iodination}

Preparation of Homopolymer Poly(vinyl benzyl iodide) (hPVBI) and Random Copolymer Poly(St-r-vinyl benzyl iodide) (r-PS-PVBI). To a solution of benzyl chloride-containing polymer $(0.94 \mathrm{~g}$ of h-PVBC, or $1.20 \mathrm{~g}$ of r-PS-PVBC) in dried acetone, a 1.2-fold molar excess of potassium iodide $(1.2088 \mathrm{~g}$; $0.3500 \mathrm{~g}$ ) was added. The reaction mixture was then stirred at $50{ }^{\circ} \mathrm{C}$ for $4 \mathrm{~h}$. Removal of acetone under vacuum yielded the iodinated polymer and excess of KI. Subsequently, $200 \mathrm{~mL}$ of saturated sodium thiosulfate solution was added to the solid mixture. The solution was then extracted with $200 \mathrm{~mL}$ of ethyl acetate three times. Precipitation was performed by adding the ethyl acetate solution into methanol. The precipitates were finally dried in vacuum for $4 \mathrm{~h}$ at $50{ }^{\circ} \mathrm{C}$ to obtain solid compound, h-PVBI or r-PS-PVBI. ${ }^{1} \mathrm{H} \mathrm{NMR}\left(200 \mathrm{MHz}, \mathrm{CDCl}_{3}\right.$, $\delta$, ppm): 1.10-2.22 (br, 6H), 4.20-4.60 (br, 2H, - $\left.\mathrm{CH}_{2}-\mathrm{I}\right), 6.10-$ 7.20 (br, 9H) ppm. FT-IR spectrum $(v): 1152 \mathrm{~cm}^{-1}\left(\mathrm{CH}_{2}-\mathrm{I}\right)$. [hPVBI: Elemental analysis (Found: C, 51.11; H, $4.48 \%$ requires C, 48.84; H, 4.07\%). $M_{\mathrm{n}}=16364$, polydispersity $=2.27$, $\left.T_{\mathrm{g}}=107^{\circ} \mathrm{C}\right]$.

[r-PS-PVBI: Elemental analysis (Found: C, 79.32; H, 6.73\% requires $\mathrm{C}, 77.90 ; \mathrm{H}, 6.49 \%) . M_{\mathrm{n}}=18901$, polydispersity = $\left.1.35, T_{\mathrm{g}}=107^{\circ} \mathrm{C}\right]$.

\section{Isocyanation}

Preparation of Poly(vinyl benzyl isocyanate) (h-PVBNCO) and Poly(St-r-vinyl benzyl isocyanate) (r-PS-PVBNCO). The iodinated polymer (1.00 $\mathrm{g}$ of h-PVBI or $0.70 \mathrm{~g}$ of r-PS-PVBI) and a 1.05-fold molar excess of $\mathrm{AgOCN}$ were mixed and stirred at $70{ }^{\circ} \mathrm{C}$ in toluene for $24 \mathrm{~h}$. The polymer solution was filtered to separate excess of AgOCN and AgI. Precipitation was performed by adding the concentrated polymer solution into $\mathrm{n}$-hexane. The precipitates were finally dried in vacuum for $4 \mathrm{~h}$ at $50{ }^{\circ} \mathrm{C}$ to obtain solid compounds, h-PVBNCO or r-PS- 
PVBNCO. ${ }^{1} \mathrm{H}$ NMR (200 MHz, $\left.\mathrm{CDCl}_{3}, \delta, \mathrm{ppm}\right): 1.10-2.22$ (br, $6 \mathrm{H}$ ), 4.20-4.60 (br, 2H, - $\mathrm{CH}_{2}-\mathrm{NCO}$ ), 6.10-7.20 (br, 9H) ppm. FT-IR spectrum $(v)$ : $2260 \mathrm{~cm}^{-1}\left(\mathrm{CH}_{2}-\mathrm{NCO}\right)$. [h-PVBNCO: Elemental analysis (Found: C, 76.11; H, 6.43; N, 5.89; O, $6.90 \%$ requires $\mathrm{C}, 73.16 ; \mathrm{H}, 5.63 ; \mathrm{N}, 6.41 ; \mathrm{O}, 7.32 \%) . T_{\mathrm{g}}=$ $\left.137^{\circ} \mathrm{C}\right]$.

[r-PS-PVBNCO: Elemental analysis (Found: C, 88.04; H, $8.07 ; \mathrm{N}, 1.33 ; \mathrm{O}, 1.78 \%$ requires $\mathrm{C}, 87.40 ; \mathrm{H}, 7.16 ; \mathrm{N}, 1.45 ; \mathrm{O}$, $\left.1.94 \%) . T_{\mathrm{g}}=111^{\circ} \mathrm{C}\right]$.

\section{Grafting HEMA on to the h-PVBNCO}

Preparation of h-Poly(2-(4-vinylbenzylcarbamoyloxy)ethyl methacrylate), h-PVBH. The h-PVBNCO (0.50g) sample was dissolved in toluene $(10 \mathrm{~mL})$ and treated with excess of HEMA $(0.61 \mathrm{~g})$ at $50^{\circ} \mathrm{C}$ for $4 \mathrm{~h}$. The polymer, h-PVBH, was then purified by precipitation from toluene to methanol. ${ }^{1} \mathrm{H}$ NMR (200 MHz, DMSO- $\left.d_{6}, \delta, \mathrm{ppm}\right): 1.10-2.22$ (br, $\left.6 \mathrm{H}\right)$, 1.75-2.00 (s, 3H), 3.50-3.75 (t, 2H, - $\left.\mathrm{CH}_{2}\right), 4.00-4.20(\mathrm{t}, 2 \mathrm{H}$, $\left.-\mathrm{CH}_{2}\right), 5.50-5.70(\mathrm{~s}, 1 \mathrm{H},-\mathrm{C}=\mathrm{C}-\mathrm{H}), 5.90-6.10(\mathrm{~s}, 1 \mathrm{H},-\mathrm{C}=\mathrm{C}-$ $\mathrm{H})$, 4.2-4.5 (br, 2H, - $\mathrm{CH}_{2}-$ ), 6.10-7.60 (br, 9H). FT-IR spectrum $(v) \mathrm{cm}^{-1}: 1678 \mathrm{~cm}^{-1}(-\mathrm{NH}-\mathrm{C}=\mathrm{O})$. [h-PVBH: Elemental analysis (Found: C, 70.99; H, 6.43; N, 3.85; O, 17.88\% requires $\mathrm{C}, 68.90 ; \mathrm{H}, 6.63 ; \mathrm{N}, 4.16 ; \mathrm{O}, 19.02 \%) . M_{\mathrm{n}}=17242$, polydispersity $\left.=1.86, T_{\mathrm{g}}=128^{\circ} \mathrm{C}\right]$.

[r-PS-PVBH: Elemental analysis (Found: C, 84.88; H, $7.44 ; \mathrm{N}, 1.37 ; \mathrm{O}, 6.44 \%$ requires $\mathrm{C}, 84.09 ; \mathrm{H}, 7.32 ; \mathrm{N}$, $1.48 ; O, 6.79 \%) . M_{\mathrm{n}}=19748$, polydispersity $=1.35, T_{\mathrm{g}}=$ $\left.102{ }^{\circ} \mathrm{C}\right]$.

\section{Measurements}

${ }^{1} \mathrm{H}$ NMR spectra were taken on a Varian Gemini-200 FT NMR spectrometer using $\mathrm{CDCl}_{3}$ and DMSO- $d_{6}$. IR measurements were performed on a Perkin-Elmer Spectrum One FT-IR spectrometer. Gel permeation chromatography (GPC) was performed in THF using a Waters Apparatus equipped with Waters Styragel columns and a refractive index detector with polystyrene calibration.

\section{RESULTS AND DISCUSSION}

\section{Synthesis and Characterization of Polymers}

In bulk living free radical polymerization, ${ }^{11-16} 1 \%$ benzoic acid was added to inhibit the thermal polymerization which is caused by the high reactivity of $\mathrm{VBC}$ monomer at high temperatures. ${ }^{17-19}$ The polydispersities of h-PVBC and r-PSPVBC can be controlled to levels under 1.46. The segment ratio (St/VBC) of r-PS-PVBC based on ${ }^{1} \mathrm{H} \mathrm{NMR}$, is 5:1 (133:26). In addition, the repeat unit number of h-PVBC is 78. ${ }^{1} \mathrm{H}$ NMR and FT-IR characterizations reveal no significant difference between h-PVBC and r-PS-PVBC. In one example, the chloromethyl group $\left(-\mathrm{CH}_{2}-\mathrm{Cl}\right)$ absorption peak of h-PVBC is located at $1266 \mathrm{~cm}^{-1}$ in the FT-IR spectrum, and at $4.44 \mathrm{ppm}$ in the ${ }^{1} \mathrm{H}$ NMR spectrum, as presented in Figures 1 and $2 \mathrm{~A}$. Moreover, the chloromethyl group $\left(-\mathrm{CH}_{2}-\mathrm{Cl}\right)$ absorption peak of r-PS-PVBC is also located at the same wavenumber and chemical shift mentioned in the h-PVBC sample.

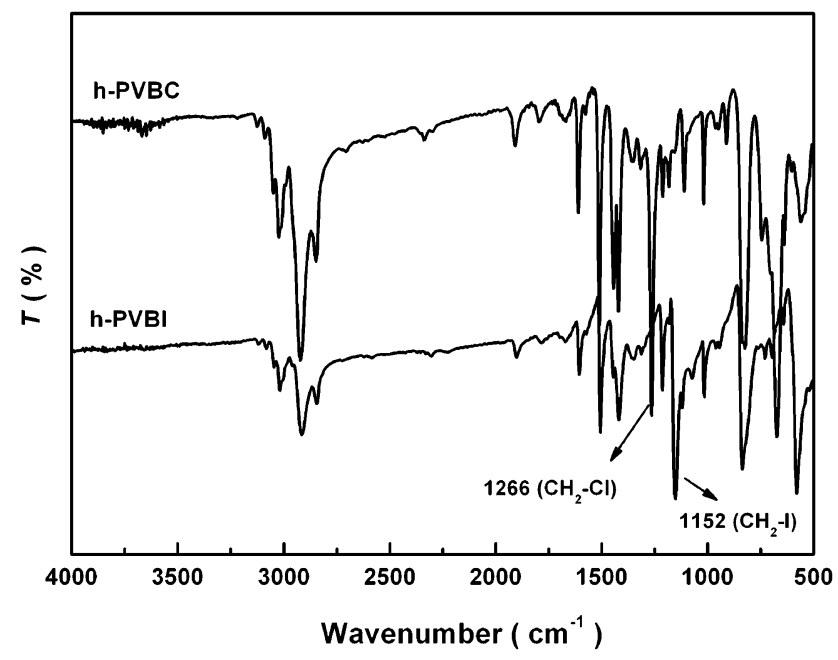

Figure 1. FT-IR spectra of h-PVBC and h-PVBI.
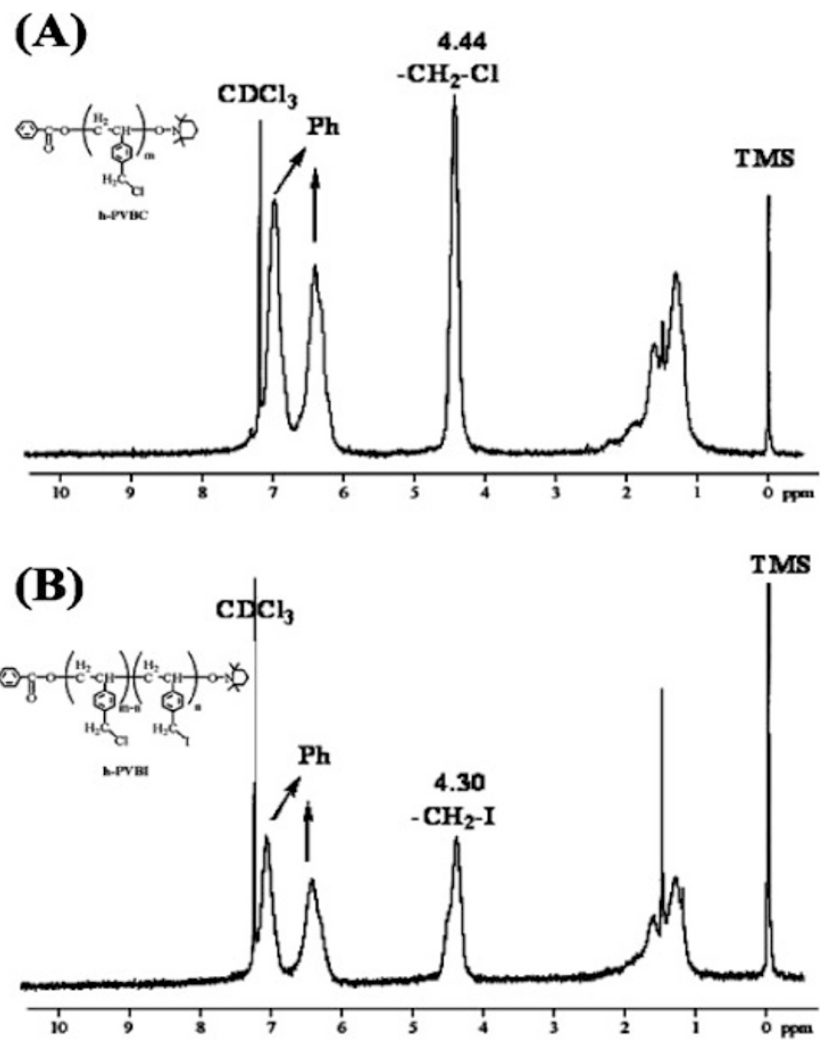

Figure 2. ${ }^{1} \mathrm{H}$ NMR spectra of (A) h-PVBC and (B) h-PVBI.

\section{Iodination}

The chloromethyl groups of h-PVBC and r-PS-PVBC were modified to iodomethyl groups by iodination. In FT-IR spectra, a weak absorption peak associated with chloromethyl groups $\left(-\mathrm{CH}_{2}-\mathrm{Cl}\right)$ appeared at $1266 \mathrm{~cm}^{-1}$, whereas a strong absorption peak associated with the iodide group $\left(-\mathrm{CH}_{2}-\mathrm{I}\right)$ was located at $1152 \mathrm{~cm}^{-1}$ after iodination substitution (Figure 1). As shown in ${ }^{1} \mathrm{H}$ NMR spectra, the peak at $4.44 \mathrm{ppm}$ was shifted to $4.30 \mathrm{ppm}$ (Figure 2). The presence of a shoulder indicates that iodination 


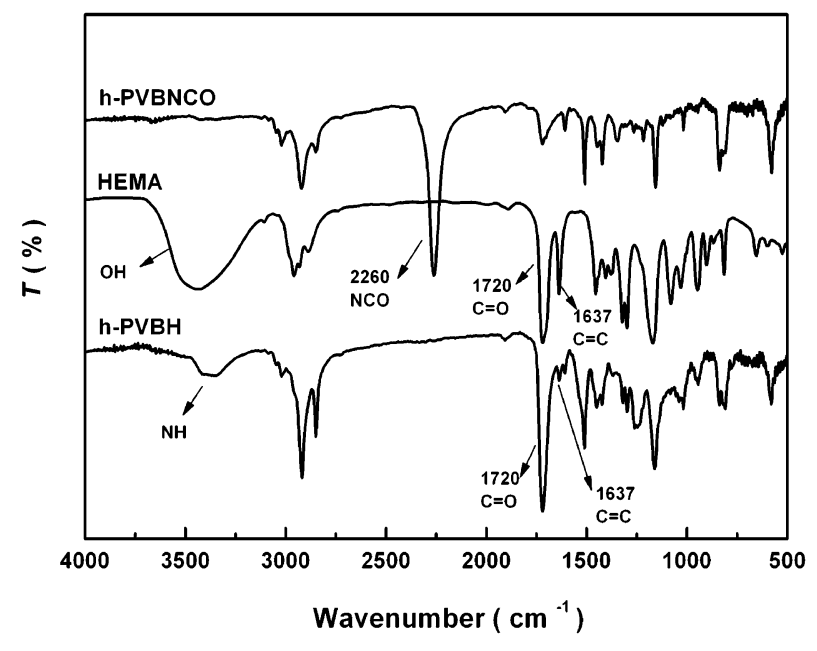

Figure 3. FT-IR spectra of h-PVBNCO, HEMA and h-PVBH.

was not complete. The h-PVBI and r-PS-PVBI conversions by iodination as calculated from ${ }^{1} \mathrm{H}$ NMR integral areas were $75 \%$ and $90 \%$, respectively. Those conversions were obtained regardless of the reactant type, sodium iodide or potassium iodide. The iodination conversion for r-PS-PVBI is higher than that for h-PVBI. This can be explained by larger steric hindrance effect in the homopolymer system. As the extent of iodination substitution increased, the steric hindrance due to the presence of the iodide ion also gradually increases. The effect of steric hindrance was more pronounced in the more crowded homopolymer system despite that the iodide ion is a good nucleophile.

\section{Isocyanation}

The h-PVBI and r-PS-PVBI polymers were reacted with silver cyanate respectively in toluene at $70^{\circ} \mathrm{C}$ and the isocyanation reaction was monitored by FT-IR. After isocyanation, the absorption peak of isocyanate group (NCO) appeared at $2260 \mathrm{~cm}^{-1}$ for h-PVBNCO (Figure 3). However, no absorption difference was found between h-PVBI and h-PVBNCO in ${ }^{1} \mathrm{H}$ NMR spectra. The chemical shifts associated with methyl iodide $\left(-\mathrm{CH}_{2}-\mathrm{I}\right)$ and methyl isocyanate $\left(-\mathrm{CH}_{2}-\mathrm{NCO}\right.$ ) were both located at $4.30 \mathrm{ppm}$ (Figure $2 \mathrm{~B}$ and Figure 4A). Gaonkar et al. ${ }^{9}$ also found that the methyl isocyanate $\left(-\mathrm{CH}_{2}-\mathrm{NCO}\right)$ of the polymer was located at $4.30 \mathrm{ppm}$, whereas the methyl isocyanate of trimer was located at $5.10 \mathrm{ppm}$. Unlike previous results, ${ }^{9}$ no byproduct (dimer or trimer) was present after isocyanation in the PVBNCO samples synthesized in this work. Table I presents the absorption characteristics of FT-IR and ${ }^{1} \mathrm{H}$ NMR for the homopolymer system.

However, the isocyanation conversion can not be decided by the ${ }^{1} \mathrm{H}$ NMR investigation. This is because no absorption difference was observed for the methyl iodide $\left(-\mathrm{CH}_{2}-\mathrm{I}\right)$ or methyl isocyanate $\left(-\mathrm{CH}_{2}-\mathrm{NCO}\right)$ in the ${ }^{1} \mathrm{H} \mathrm{NMR}$ spectra. Gaonkar et al. ${ }^{9}$ synthesized carbamate by treating h-PVBNCO with methanol, and calculated the conversion by integrating areas under the ${ }^{1} \mathrm{H}$ NMR spectra. This approach is not feasible
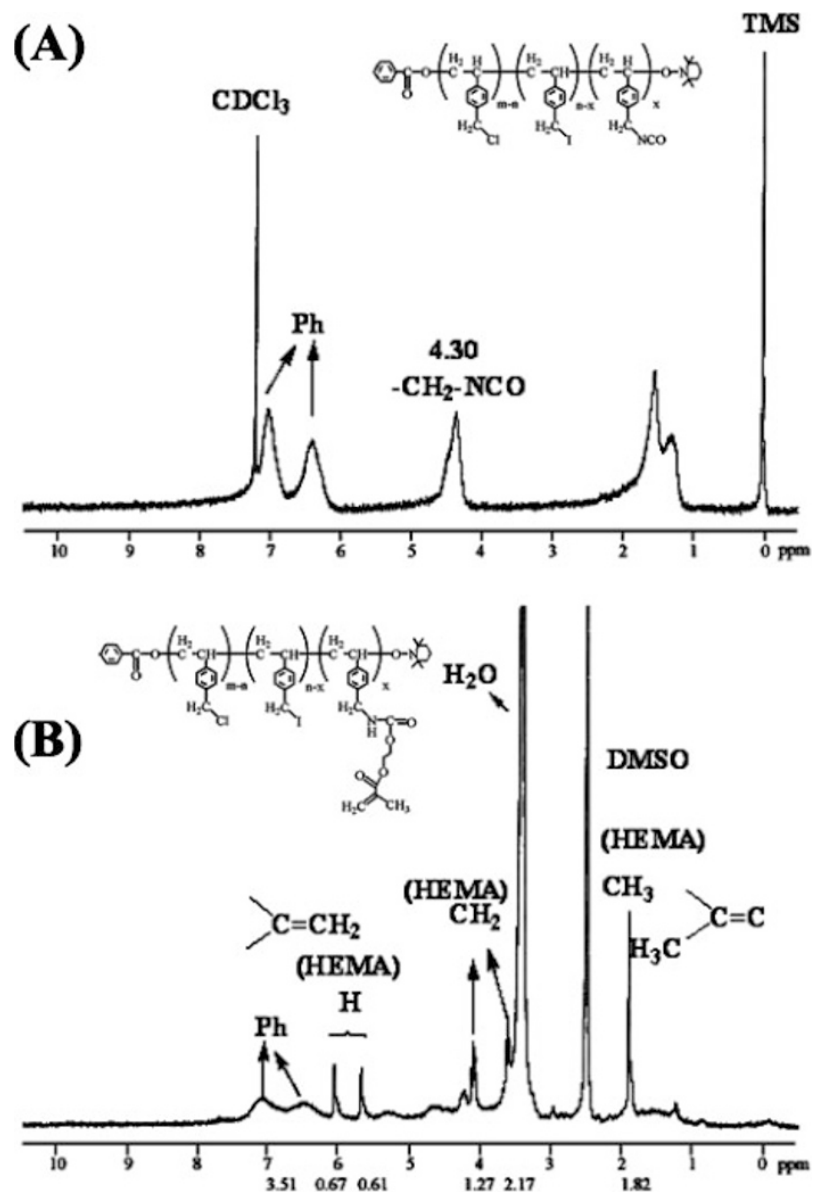

Figure 4. ${ }^{1} \mathrm{H}$ NMR spectra of (A) h-PVBNCO and (B) h-PVBH.

Table I. Absorption characteristics of FT-IR and ${ }^{1} \mathrm{H}$ NMR for the homopolymer system

\begin{tabular}{ccc}
\hline & IR $\left(\mathrm{cm}^{-1}\right)$ & NMR $(\mathrm{ppm})$ \\
\hline h-PVBC & 1266 & $4.44^{\mathrm{a}}$ \\
h-PVBI & 1152 & $4.30^{\mathrm{a}}$ \\
h-PVBNCO & 2260 & $4.30^{\mathrm{a}}$ \\
h-PVBH & 1637 & $5.66,6.04^{\mathrm{b}}$ \\
\hline
\end{tabular}

${ }^{\mathrm{a}}$ In $\mathrm{CDCl}_{3} .{ }^{\mathrm{b}}$ In DMSO- $d_{6}$.

in this work because the conversion was higher than that in Gaonkar's system. When the polymers possessed a large amount of polar carbamate functional groups, the solubility became poor. The carbamate-containing polymers in this work could not be dissolved in any medium. Therefore, HEMA was utilized to replace methanol to graft on the h-PVBNCO, as shown in Scheme 2. Three reasons for choosing HEMA to be grafted onto h-PVBNCO are (1) HEMA is liquid at room temperature and the excessive HEMA can be easily removed after grafting, (2) HEMA-grafted h-PVBNCO is much more soluble in organic solvents than methanol-grafted one, and (3) more importantly, the HEMA-containing polymer could be used for photo-crosslinking. ${ }^{20,21}$

As shown in Figure 3, the absorption from the isocyanate functional groups was absent at $2260 \mathrm{~cm}^{-1}$ while those from $\mathrm{N}$ - 


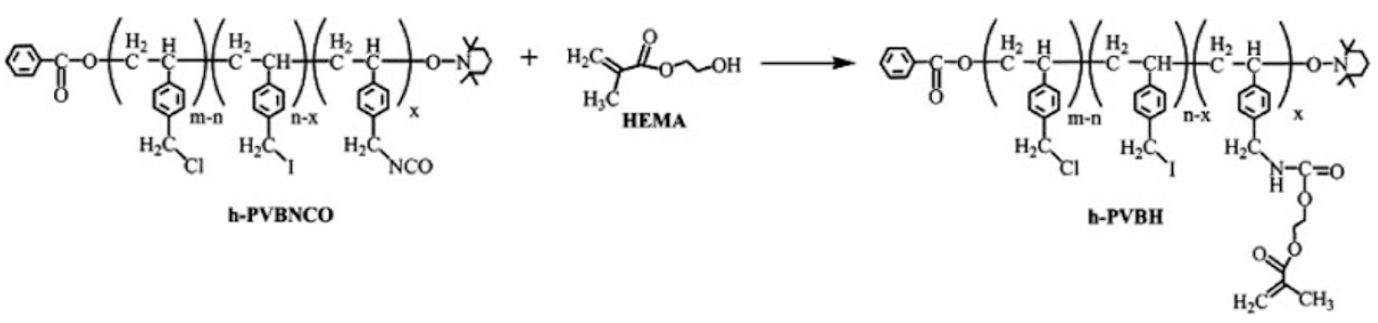

Scheme 2. Synthesis of h-PVBH.

Table II. Conversion of iodination and isocyanation from starting materials h-PVBC and r-PS-PVBC

\begin{tabular}{|c|c|c|c|}
\hline \multirow{2}{*}{$\begin{array}{l}\text { Starting } \\
\text { material }\end{array}$} & \multicolumn{3}{|c|}{ Conversion (\%) } \\
\hline & $\begin{array}{l}\text { lodination } \\
(\mathrm{Cl} \rightarrow \mathrm{I})\end{array}$ & $\begin{array}{l}\text { Isocyanation } \\
(I \rightarrow \text { NCO })\end{array}$ & $\begin{array}{l}\text { Total conversion } \\
\quad(\mathrm{Cl} \rightarrow \mathrm{NCO})\end{array}$ \\
\hline h-PVBC 78 & $\begin{array}{c}75 \% \\
\mathrm{PVBC}_{19}-\mathrm{PVBI}_{59}\end{array}$ & $\begin{array}{c}97 \% \\
\mathrm{PVBC}_{19}-\mathrm{PVBI}_{2}-\mathrm{PVBNCO}_{57}\end{array}$ & $73 \%$ \\
\hline $\mathrm{r}-\mathrm{PS}_{133}-\mathrm{PVBC} \mathrm{C}_{26}$ & $\begin{array}{c}90 \% \\
r-\mathrm{PS}_{133}-\mathrm{PVBC}_{2}-\mathrm{PVBI}_{24}\end{array}$ & $\begin{array}{c}90 \% \\
r-\mathrm{PS}_{133}-\mathrm{PVBC}_{2}-\mathrm{PVBI}_{2}-\mathrm{PVBNCO}_{22}\end{array}$ & $81 \%$ \\
\hline
\end{tabular}

$\mathrm{H}, \mathrm{C}=\mathrm{O}$ and $\mathrm{C}=\mathrm{C}$ bonds of h-PVBH were located at $3300 \mathrm{~cm}^{-1}, 1720 \mathrm{~cm}^{-1}$, and $1636 \mathrm{~cm}^{-1}$, respectively. As shown in Figure 4, the isocyanation conversion was then determined by calculating the proton area ratio between the alkene $\left(\mathrm{C}=\mathrm{CH}_{2}\right)$ functional groups from HEMA and from aromatic functional groups of the polymer. After grafting, the theoretical ratio of the integral areas under the absorption peaks associated with $\mathrm{H}: \mathrm{CH}_{2}: \mathrm{CH}_{3}$ of HEMA is 2:4:3. As shown in Figure 4, the experimental ratio $\left(\mathrm{H}: \mathrm{CH}_{2}: \mathrm{CH}_{3}\right)$ is $(0.61+0.67):(1.27 \times 2)$ : (1.82), which matches the theoretical ratio. When the iodination and isocyanation conversions are both $100 \%$, the ratio of protons from aromatic ring to protons from alkene is $2: 1$. Based on the above, the total conversion from h-PVBC to h-PVBNCO was $73 \%((0.61+0.67) \times 2 / 3.51)$. As mentioned in previous section, the conversion from $\mathrm{h}-\mathrm{PVBC}$ to $\mathrm{h}-\mathrm{PVBI}$ is $75 \%$. Therefore, the isocyanation conversion, i.e., from h-PVBI to hPVBNCO would reach $97 \%(0.73 / 0.75)$, calculated from the ratio of overall isocyanation conversion (from h-PVBC to $h-$ PVBNCO) to iodination conversion (from h-PVBC to h-PVBI). In addition, the chemical shift of methyl iodide was located at $4.30 \mathrm{ppm}$ originally. After grafting of HEMA, a chemical shift was at $4.22 \mathrm{ppm}$ corresponding to methylene of benzyl group indicating the formation of carbamate functional group. This two absorption peaks can be clearly distinguished from the NMR spectra in Figure 4. Similar results were also reported in our previous researches. ${ }^{22,23}$ Therefore, the isocyanation conversion from h-PVBI to h-PVBNCO would reach $97 \%$ and from r-PS-PVBI to r-PS-PVBNCO would reach $90 \%$. Total conversions from the chlorinated polymers to h-PVBNCO and r-PS-PVBNCO were $73 \%$ and $81 \%$, respectively. Table II shows reaction conversions of iodination and isocyanation from starting materials h-PVBC and r-PS-PVBC. This is much higher than those of the previous studies. ${ }^{8,9}$ Number average molecular weights and PDIs for the polymers are shown in Table III. For random copolymer system, after iodination or isocyanation, the $M_{\mathrm{n}}$ and PDI increased slightly. This is
Table III. GPC data for polymers

\begin{tabular}{ccc}
\hline Sample & $M_{\mathrm{n}}$ & PDI $^{\mathrm{a}}$ \\
\hline h-PVBC & 12140 & 1.46 \\
h-PVBI & 16364 & 2.27 \\
h-PVBH & 17242 & 1.86 \\
r-PS-PVBC & 17885 & 1.21 \\
r-PS-PVBI & 18901 & 1.35 \\
r-PS-PVBH & 19748 & 1.35 \\
\hline
\end{tabular}

a Determined by GPC analysis in THF.

because the functionalization content $(26 / 159)$ is low and the functional groups scatter loosely in the PS matrices. However, the variation in $M_{\mathrm{n}}$ and PDI for iodination or isocyanation is obvious in homopolymer system. After iodination of the hPVBC, the large iodide ion replacing chloride ion onto the polymer side chain would result in higher $M_{\mathrm{n}}$ and broader PDI. Moreover, the PDI decreased to 1.86 and the $M_{\mathrm{n}}$ increased slightly after the introduction of flexible HEMA onto the polymer side chain.

It is important to note that a strong absorption peak of isocyanate functional group was detected by FT-IR after $1.5 \mathrm{~h}$ of isocyanation. The conversion would reach $90 \%$ after $24 \mathrm{~h}$ reaction time. The isocyanation conversion is highly dependent on the reaction time as shown in Figure 5. Without the addition of catalyst and yielding of trimer or dimer byproduct, the strategy to synthesize high conversion isocyanato polymers from the iodinated polymers was successful.

However, the isocyanato polymers could not be obtained by treating PVBCs (including h-PVBC and r-PS-PVBC) with silver cyanate, sodium cyanate or potassium cyanate, as shown in Scheme 1 (route III). This is because the chloride ion is not a good leaving group. Similarly, PVBIs (including h-PVBI and r-PS-PVBI) did not yield isocyanato polymers when reacted with sodium cyanate or potassium cyanate, as shown in Scheme 1 (route IV). When the primary methyl iodide was 


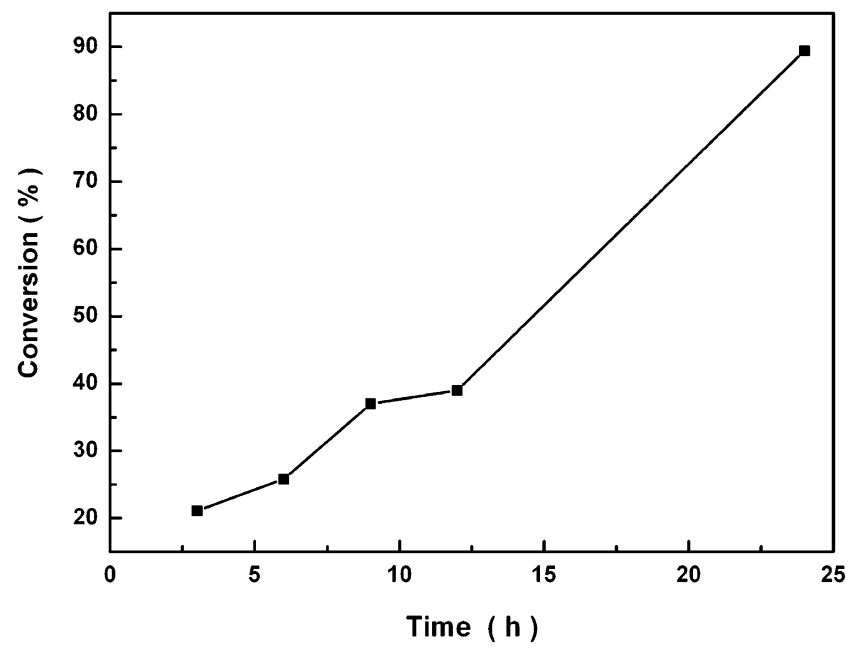

Figure 5. Conversion of isocyanation for $\mathrm{h}-\mathrm{PVBI}$ at $70^{\circ} \mathrm{C}$ as a function of reaction time.

Table IV. Thermal properties for polymers

\begin{tabular}{cccccc}
\hline Type & Code & Repeat unit & $T_{\mathrm{g}}{ }^{\mathrm{a}}\left({ }^{\circ} \mathrm{C}\right)$ & $T_{\mathrm{d}}{ }^{\mathrm{b}}\left({ }^{\circ} \mathrm{C}\right)$ & Char Yield $(\%)$ \\
\hline Homopolymer & h-PS & 157 & 95 & 313 & 0 \\
\hline \multirow{5}{*}{ Homopolymer } & h-PVBC & & 98 & 350 & 30 \\
& h-PVBI & & 107 & 239 & 18 \\
& h-PVBNCO & 78 & 137 & 293 & 48 \\
& h-PVBH & & 128 & 306 & 40 \\
\hline \multirow{4}{*}{ Random } & r-PS-PVBC & & 102 & 348 & 6 \\
copolymer & r-PS-PVBI & & 107 & 262 & 3 \\
& r-PS-PVBNCO & $133: 26$ & 111 & 313 & 10 \\
& r-PS-PVBH & & 102 & 266 & 7 \\
\hline
\end{tabular}

${ }^{a}$ Data was taken from DSC second heating trace, with a heating rate of $10^{\circ} \mathrm{C} / \mathrm{min}$. ${ }^{\text {b Data }}$ was taken from TGA trace, $5 \%$ weight loss.

treated with silver cyanate, silver iodide would precipitate from toluene. This served as a driving force to promote the isocyanation conversion on the basis of Le Chatelier's Principle. In contrast to the silver cyanate system, the sodium iodide is still ionic and soluble in the medium. However the iodide ion is still a good nucleophile and would favor to react with the primary methyl cation. This means that the iodide ion prefers serving as a nucleophile to act as a leaving group in the sodium cyanate system. This strategy was also adopted in the synthesis of bi-functional monomer, vinyl benzyl isocyanate from vinyl benzyl chloride. High yields in iodination and isocyanation were also achieved.

\section{Thermal Properties}

Table IV presents thermal properties of the polymers. For the homopolymer system (h-PVBC; the number of repeating units: 78), the $T_{\mathrm{g}}$ is $98^{\circ} \mathrm{C}$. After iodination of the homopolymer, the $T_{\mathrm{g}}$ of h-PVBI increased to $107^{\circ} \mathrm{C}$ due to the presence of a strong electronegative iodine group. However, the iodide functional groups are not thermally stable, and at high temperatures readily decompose and release hydrogen iodide or $\mathrm{I}_{2}$. Once the iodide functional groups had been functionalized in the polymer, the $T_{\mathrm{g}}$ of iodide-containing polymer remained high but the $T_{\mathrm{d}}$ was low. The char yield was decreased to $18 \%$. As the polymer was isocyanated, the $T_{\mathrm{g}}$ was increased to $137^{\circ}$ due to the presence of strong polar functional groups in the h-PVBNCO. At $800^{\circ} \mathrm{C}$, the char yield was $48 \%$. This means that the isocyanation process is successful and the crosslinking of isocyanate functional groups occurred at high temperatures. High char yields were also observed for the h-PVBH system because of the presence of crosslinking reactions. However, the crosslinking reactions were derived from vinyl groups by heating process. After grafting HEMA to the polymer, $T_{\mathrm{g}}$ of HEMA-containing polymer was about $128{ }^{\circ} \mathrm{C}$, whereas the char yield was around $40 \%$.

Although the isocyanate group and vinyl group were active functional groups at high temperatures, the char yields for random copolymer systems were low (under 10\%). In the random copolymer, the polystyrene is a major component, which can effectively isolate the functionalized styrene units and prevent them from reacting with each other. Therefore, high char yield was absent without crosslinking reactions. In general, these random copolymers possessed only one $T_{\mathrm{g}}$ even though they were composed of different segments, as shown in the Table IV. The polar isocyanate group and hydrogen-bondingcontaining HEMA were present in various random copolymers, respectively. However, variations of $T_{\mathrm{g}}$ for these random copolymers were not as significant as those of the homopolymer systems. Due to the presence of the major component, styrene segments in the random copolymer system, the thermal properties of random copolymers were dominated by the PS segments. The $T_{\mathrm{g}} \mathrm{s}$ of r-PS-PVBNCO and r-PS-PVBH can be predicted using Fox's equation: $1 / T_{\mathrm{g}}=M_{1} / T_{\mathrm{g} 1}+M_{2} / T_{\mathrm{g} 2} .{ }^{24}$ The predicted values were $104{ }^{\circ} \mathrm{C}$ and $106^{\circ} \mathrm{C}$ while the experimental values were $111^{\circ} \mathrm{C}$ and $102^{\circ} \mathrm{C}$ for r-PS-PVBNCO and r-PS$\mathrm{PVBH}$, respectively. The small deviations of $6.3 \%$ for r-PSPVBNCO and $3.92 \%$ for r-PS-PVBH indicate that Fox's equations are reasonably accurate in our systems.

\section{CONCLUSION}

Anti-yellowing aliphatic isocyanate polymers with narrow polydispersities have been synthesized via iodination of hPVBC and r-PS-PVBC, and subsequent isocyanation of $h-$ PVBI and r-PS-PVBI. This approach is highly selective at high conversion and yields no dimer, trimer or any other byproducts. This high selectivity is due to the fact that the iodide ion is not only a good nucleophile for iodination but also a good leaving group for the isocyanation that follows. Through the iodination synthetic route, high conversion isocyanato polymers can be obtained. More importantly, a bi-functional monomer, VBNCO, can be synthesized through this synthetic strategy with high yields. A more thorough investigation in this system and its applications is being pursued.

Acknowledgment. Partial financial support from National Science Council of Taiwan ROC is gratefully acknowledged. This work is also supported in part by the Ministry of Education, Taiwan under ATU plan. 
Received: June 1, 2009

Accepted: August 9, 2009

Published: September 16, 2009

\section{REFERENCES}

1. A. Mahajan, S. R. Chhabra, and W. C. Chan, Tetrahedron Lett., 40, 4909 (1999).

2. R. J. Booth and J. C. Hodges, J. Am. Chem. Soc., 119, 4882 (1997).

3. G. H. Hu, H. Li, L. F. Feng, and L. A. Pessan, J. Appl. Polym. Sci., 88, 1799 (2003).

4. S. Mohammed, E. S. Daniels, A. Klein, and M. S. El-Aasser, J. Appl. Polym. Sci., 67, 559 (1998).

5. D. D. Wisnoski, W. H. Leister, K. A. Strauss, Z. Zhao, and C. W. Lindsley, Tetrahedron Lett., 44, 4321 (2003).

6. K. R. Yoon and S. Lee, J. Macromol. Sci., Part B: Phys., 45, 859 (2006).

7. L. Barner, S. Perera, S. Sandanayake, and T. P. Davis, J. Polym. Sci., Part A: Polym. Chem., 44, 857 (2006).

8. M. C. Himel, M. Park, and L. M. Richard, U. S. Patent 2866801 (1958).

9. S. R. Gaonkar, K. Srinivasan, and G. S. Kumar, J. Polym. Sci., Part A: Polym. Chem., 30, 1911 (1992).

10. A. Holm and C. Wentrup, Acta Chem. Scand., 20, 2123 (1966).

11. C. J. Hawker, J. Am. Chem. Soc., 116, 11185 (1994).
12. C. J. Hawker and J. L. Hedrick, Macromolecules, 28, 2993 (1995).

13. C. J. Hawker, Acc. Chem. Res., 30, 373 (1997).

14. M. K. Georges, R. P. N. Veregin, P. M. Kazmaier, and G. K. Hamer, Macromolecules, 26, 2987 (1993).

15. A. Goto and T. Fukuda, Macromol. Chem. Phys., 201, 2138 (2000).

16. M. Baumann and G. Schmidt-Naake, Macromol. Chem. Phys., 202, 2727 (2001).

17. W. Devonport, L. Michalak, E. Malmström, M. Mate, B. Kurdi, C. J. Hawken, G. G. Barclay, and R. Sinta, Macromolecules, 30, 1929 (1997).

18. P. M. Kazmaier, K. Daimon, M. K. Georges, G. K. Hamer, and R. P. N. Veregin, Macromolecules, 30, 2228 (1997).

19. T. Tsoukatos, S. Pispas, and N. Hadjichristidis, Macromolecules, 33, 9504 (2000).

20. D. A. Wang, C. G. Williams, Q. Li, B. Sharma, and J. H. Elisseeff, Biomaterials, 24, 3969 (2003).

21. R. W. Arcís, A. López-Macipe, M. Toledano, E. Osorio, R. Rodríguez-Clemente, J. Murtra, M. A. Fanovich, and C. D. Pascual, Dent. Mater., 18, 49 (2002).

22. W. H. Ting, S. A. Dai, Y. F. Shih, I. K. Yang, W. C. Su, and R. J. Jeng, Polymer, 49, 1497 (2008).

23. W. H. Ting, C. C. Chen, S. A. Dai, S. Y. Suen, I. K. Yang, Y. L. Liu, F. M. C. Chen, and R. J. Jeng, J. Mater. Chem., 19, 4819 (2009).

24. L. H. Sperling, "Introduction to physical polymer science," 2nd ed., Wiley, New York, 1992. 